\title{
Worm Phenotype Ontology: Integrating phenotype data within and beyond the C. elegans community
}

\author{
Gary Schindelman ${ }^{2+}$, Jolene S Fernandes ${ }^{2 \dagger}$, Carol A Bastiani ${ }^{2 \dagger}$, Karen Yook ${ }^{2}$, Paul W Sternberg ${ }^{1,2^{*}}$
}

\begin{abstract}
Background: Caenorhabditis elegans gene-based phenotype information dates back to the 1970's, beginning with Sydney Brenner and the characterization of behavioral and morphological mutant alleles via classical genetics in order to understand nervous system function. Since then C. elegans has become an important genetic model system for the study of basic biological and biomedical principles, largely through the use of phenotype analysis. Because of the growth of $C$. elegans as a genetically tractable model organism and the development of large-scale analyses, there has been a significant increase of phenotype data that needs to be managed and made accessible to the research community. To do so, a standardized vocabulary is necessary to integrate phenotype data from diverse sources, permit integration with other data types and render the data in a computable form.

Results: We describe a hierarchically structured, controlled vocabulary of terms that can be used to standardize phenotype descriptions in C. elegans, namely the Worm Phenotype Ontology (WPO). The WPO is currently comprised of 1,880 phenotype terms, $74 \%$ of which have been used in the annotation of phenotypes associated with greater than 18,000 C. elegans genes. The scope of the WPO is not exclusively limited to C. elegans biology, rather it is devised to also incorporate phenotypes observed in related nematode species. We have enriched the value of the WPO by integrating it with other ontologies, thereby increasing the accessibility of worm phenotypes to non-nematode biologists. We are actively developing the WPO to continue to fulfill the evolving needs of the scientific community and hope to engage researchers in this crucial endeavor.

Conclusions: We provide a phenotype ontology (WPO) that will help to facilitate data retrieval, and cross-species comparisons within the nematode community. In the larger scientific community, the WPO will permit data integration, and interoperability across the different Model Organism Databases (MODs) and other biological databases. This standardized phenotype ontology will therefore allow for more complex data queries and enhance bioinformatic analyses.
\end{abstract}

\section{Background}

Phenotypes are the observable physical or biochemical traits manifested by an organism in response to their genetics and environment. Phenotype designation has long been the mainstay for geneticists, allowing scientists to infer gene function from the phenotypes and genetic properties of mutations [1-3]. As methods for analyzing gene function continue to evolve, identifying

\footnotetext{
* Correspondence: pws@caltech.edu

† Contributed equally

${ }^{1}$ Howard Hughes Medical Institute, California Institute of Technology,

Pasadena, CA 91125, USA

Full list of author information is available at the end of the article
}

and characterizing phenotypes necessarily requires a means to organize phenotype information into a unified vocabulary that will allow researchers to realize that seemingly disparate gene activities may actually be affecting a similar biological process. With the publication of an essentially complete genome sequence in 1998 [4] and the definition of a complete gap-free sequence in 2005 [5], virtually every gene in C. elegans became accessible to functional analysis based on phenotypes via reverse genetics [6]. As a consequence, information from classical genetics is now complemented by high-throughput RNAi screens, individual RNAi experiments, and gene knockout data [7-9]. It has been estimated that $79 \%$ of

\section{Biomed Central}


C. elegans protein-coding genes have a known protein motif, and $21 \%$ of them have non-nematode orthologs [10]; hence, the ability to functionally and molecularly characterize these protein products is of profound consequence to the scientific field outside of the C. elegans community.

C. elegans is amenable to various other methods of genetic or physical manipulation, and the phenotypic outcomes of these modifications are also useful to discern gene function. These manipulations include transgene overexpression, cell ablation, pharmacological treatment, and genetic mosaic analysis. In addition, the phenotypic consequence of multiple modifications within the same strain describes a genetic interaction, instrumental in defining whether genes act in parallel or intersecting genetic pathways [11].

WormBase (http://www.wormbase.org) serves as a repository for the wealth of phenotype data in C. elegans. The primary literature serves as the main source for phenotype data, but WormBase also receives information from individual researchers and gene knockout consortiums through direct submissions to the database $[8,9]$. The vocabulary used to describe similar or identical phenotypes, as well as the level of descriptive resolution, often varies between these sources. Prior to July 2006, mutant alleles in WormBase (version WS160) were annotated using a free-text format. Consequently, different words were used to annotate the same phenotypes and there was no inherent hierarchical organization in the descriptions, thus making retrieval of phenotypic information via searches more cumbersome. Perhaps more importantly, although free-text data is accessible by humans, it is not readily available in a computable form and therefore hinders the ability to perform standard bioinformatic operations such as term enrichment analyses or the clustering of annotations. Lack of structure also makes it difficult and time consuming to draw effective comparisons within and between different organisms.

As a step towards overcoming these challenges, we initially developed a phenotype vocabulary limited to 127 phenotype terms in WormBase. These terms were mainly the 3-letter phenotype descriptors familiar among C. elegans researchers; such as 'Dpy' (dumpy), 'Unc' (uncoordinated), and 'Bli' (blistered). This vocabulary did not contain phenotype term definitions or references, and there was redundancy within these terms (for example both 'Prz' and 'Prl' stood for paralyzed). We developed this initial vocabulary mainly to accommodate the rapidly ballooning influx of phenotype data from large-scale RNAi experiments, and continued to use free-text descriptions for alleles. Compared to phenotypes that describe the outcome of RNAi analyses, mutant allele descriptions are typically more variable, as they have accumulated over a longer period of time, tend to be more granular (specific), and arise from many independent studies. We quickly realized that to integrate the massive amount of phenotype data across different sources, permit integration with other data types, and render the data computationally accessible, a controlled vocabulary unifying identical, similar, and related concepts was essential for optimal synthesis of accumulated data within and beyond the C. elegans research community.

With the above objectives in mind, we built the Worm Phenotype Ontology (WPO) to organize and classify phenotype data in C. elegans, utilizing ontology structure and rules set up by some of the other model organism databases (MODs) [12-14]. An ontology is a controlled vocabulary organized in a hierarchical-structure intended to represent relationships between human-interpretable concepts. Moreover, because an ontology uses a controlled vocabulary with strict relationships between its terms, it is computer-comprehensible and thus allows for complex data queries [15]. Ontologies have also proven to be powerful tools for curation consistency, as well as cross-species comparison of biological data $[12-14,16]$. Perhaps the best example of how the use of ontologies has aided in the dissemination and integration of research data across many fields is found in the widespread use of the Gene Ontology (GO) [16].

Many organism-specific ontologies are however primarily designed to cater to the needs of their individual user communities and are not mutually interoperable. The absence of cross-operability makes it extremely challenging for users to merge existing genotype-phenotype annotations from the different organism databases and compare data across species. Integration of phenotype data thus depends on the existence of cross-products with other ontologies, a goal that is facilitated by the PATO project at the OBO Foundry (http://obofoundry.org/wiki/ index.php/PATO:About). PATO-facilitated cross-products enable approximate equivalence mappings with independent phenotype ontologies developed for different organisms [17]. For example, the Mammalian Phenotype Ontology term 'Spherocytosis' can be expressed as being equivalent to the cross-products of the terms 'Erythrocyte' and 'Spherical' from the OBO Cell ontology and PATO, respectively [17]. The creation of equivalence mappings is therefore a way of linking concepts from different ontologies so users of a particular ontology can access comparative information from other ontologies.

Although the Worm Phenotype Ontology (WPO) was initially aimed at the curation of C. elegans phenotypes, we have extended its application to include phenotypic data from other nematode species. This expansion mirrors the recent evolution of WormBase to include the complete genomic sequence, gene predictions and orthology assignments from a range of nematodes [18]. Furthermore, we have increased the comparative value 
of the WPO by generating equivalence mappings for individual process-oriented phenotype terms to GO terms, thereby promoting cross-operability across different biological databases. Therefore, the organized framework of the WPO will provide non-nematode biologists with a means to interact with WormBase curated data. Finally, a structured machine readable vocabulary will allow complex data queries, expediting the identification of genes that act in the same processes or pathways, ultimately across organisms, and thus conserve valuable researcher time, effort and resources.

\section{Results}

Structure of the WPO

The overall hierarchy of WormBase's phenotype ontology mirrors the rationale and organization employed by the Gene Ontology (GO) consortium (http://www.geneontology.org) as a directed acyclic graph (DAG). Terms represent phenotype classes, and a child term represents a subclass of its parent term. Child terms in the phenotype ontology all hold an 'is_a' relationship with their parent terms. The is_a relationship is transitive, implying that if 'Phenotype $A$ ' is_a 'Phenotype B', and 'Phenotype B' is_a 'Phenotype C', then 'Phenotype A' is_a 'Phenotype C.' The hierarchical structure thus allows phenotype annotation to be made at a granular level while preserving the association of child terms to a more general parent term.

The Worm Phenotype Ontology contains one root term, 'Variant', with five direct descendants (children) (see Figure 1a). The root term, 'Variant', reflects the fact that the "control" animal for an experiment is defined as

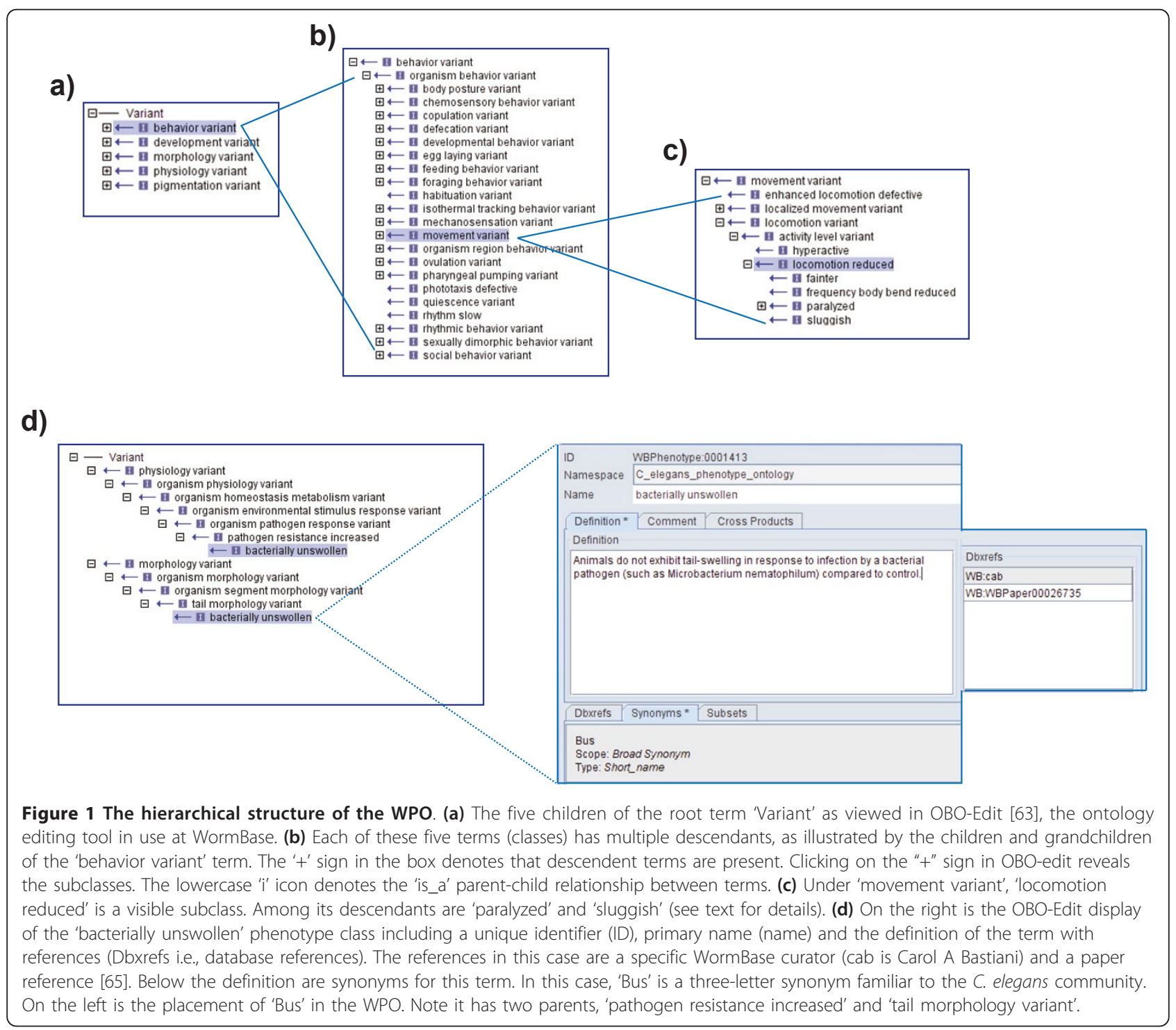


a reference strain, with characteristic properties. A phenotype that differs from the phenotype of the reference strain is defined as 'Variant.' The five direct children of 'Variant' encompass the major classes of phenotypes in C. elegans and other nematodes: 'behavior variant', 'development variant', 'morphology variant', 'physiology variant' and 'pigmentation variant'. As shown in Figure 1a, the relationship between 'Variant' and 'behavior variant' is an is_a relationship where 'Variant' is the parent and 'behavior variant' is the child. Therefore the children of 'behavior variant', such as 'organism behavior variant' are grandchildren of 'Variant' (see Figure 1b).

A phenotype term (also referred to as a primary name or name) is assigned a unique 'WBPhenotype' identifier; for example, 'bacterially unswollen' is WBPhenotype:00001413. Each phenotype term is also associated with a definition, references and synonyms (where appropriate). Synonyms are assigned so as to allow for non-uniform community jargon to denote the same type of entity without compromising efficacy and accuracy of term nomenclature. References can include the primary literature, GO term definitions or WormBase curators (see Figure 1d). To researchers both inside and outside the C. elegans community the term 'bacterially unswollen' may not be familiar; however, the placement of the terms in the ontology reflects the biology. The organization is such that a non-C. elegans researcher can browse the ontology without any prior knowledge of C. elegans biology or jargon. For example, the 'bacterially unswollen' phenotype, which describes worms that do not exhibit a tail-swelling response to infection by a bacterial pathogen, is a descendant in both the 'physiology variant' and 'morphology variant' branches. In terms of physiology, it is related to pathogen resistance; in terms of morphology it is related to tail morphology (see Figure 1d). The DAG structure permits individual terms to be children of multiple, broader parent terms.

There are now a total of 1880 phenotype terms in the WPO, all of which are defined along with their respective references. $74 \%$ of the terms in the ontology are used in annotation. Of the remaining $26 \%$ of unused terms, many of these provide structure for more granular terms that were considered necessary to capture the appropriate level of detail for a phenotype annotation so as to reflect its description in the literature. Unused phenotype terms are expected to be used as phenotype annotations in WormBase continue to grow. The current usage of phenotype terms annotated to genes is summarized in Figure 2. $12 \%$ of terms have two or more parents (number of terms with multiple paths) as multiple inheritance relationships allow us to capture the different biological contexts of a phenotype within the overall hierarchical structure [19] (See the 'bacterially unswollen' example in Figure 1d). The WPO is in compliance with The OBO Foundry principles (http://www.obofoundry.org/crit.shtml), making it a ready source for ontology users from other fields. Currently, The Biological General Repository for Interaction Datasets (BioGRID) database is utilizing the WPO for the annotation of phenotypes related to genetic interactions in C. elegans [20].

\section{Development of the WPO}

Initially we evaluated the preexisting 127 phenotype classes in WormBase and placed them within the five major classes of phenotypes in the WPO. Related terms were relegated to an appropriate location within the same branch of the hierarchy. For example, 'paralyzed' and 'sluggish' ('Slu') are both descendants of the 'locomotion reduced' branch of the ontology found in the 'behavior variant' section (see Figure 1c). Also, terms judged to be equivalent were merged (for example 'Prl' and 'Prz' for 'paralyzed'). When terms were merged, original names were maintained as synonyms, and synonyms were linked to relevant papers or curators so that the source for the names can be tracked.

The development of the WPO has been driven by curation of the primary literature; specifically, we prioritize the creation of terms based on need. One example of how a branch of the ontology becomes more refined through curation can be found in the 'dauer induction variant' branch (see Figure 3a). The earlier versions of the WPO lacked child terms for 'dauer induction variant', yet there are multiple ways to induce dauer formation in C. elegans: starvation, temperature change, pheromone application and sterol application. As we read papers pertaining to dauer biology this branch of the ontology became more refined. In addition to the papers we actively curate we also use Textpresso (http:// www.textpresso.org, an information extraction tool for biological literature) [21], WormAtlas (http://www.wormatlas.org, a site dedicated to worm anatomy), and WormBook (http://www.wormbook.org, a source of biological process reviews) [22] to help create and refine terms, definitions and synonyms. As the WPO becomes more encompassing for a particular biological phenomenon terms are added less frequently.

We also actively solicit expert input from members of the research community to create and define terms and to ensure that the ontology reflects the biology of C. elegans. Terms that describe early embryonic lethal phenotypes ('early emb') were developed in this fashion. As a result, embryonic lethal descendent terms represent the most robust, and intricate portion of the WPO (see Figure 3b). There are 116 embryonic lethal terms, developed initially by integrating and ordering phenotypes described in publications where the researchers performed high-throughput RNAi screens [23-27]. Extensive development of this 


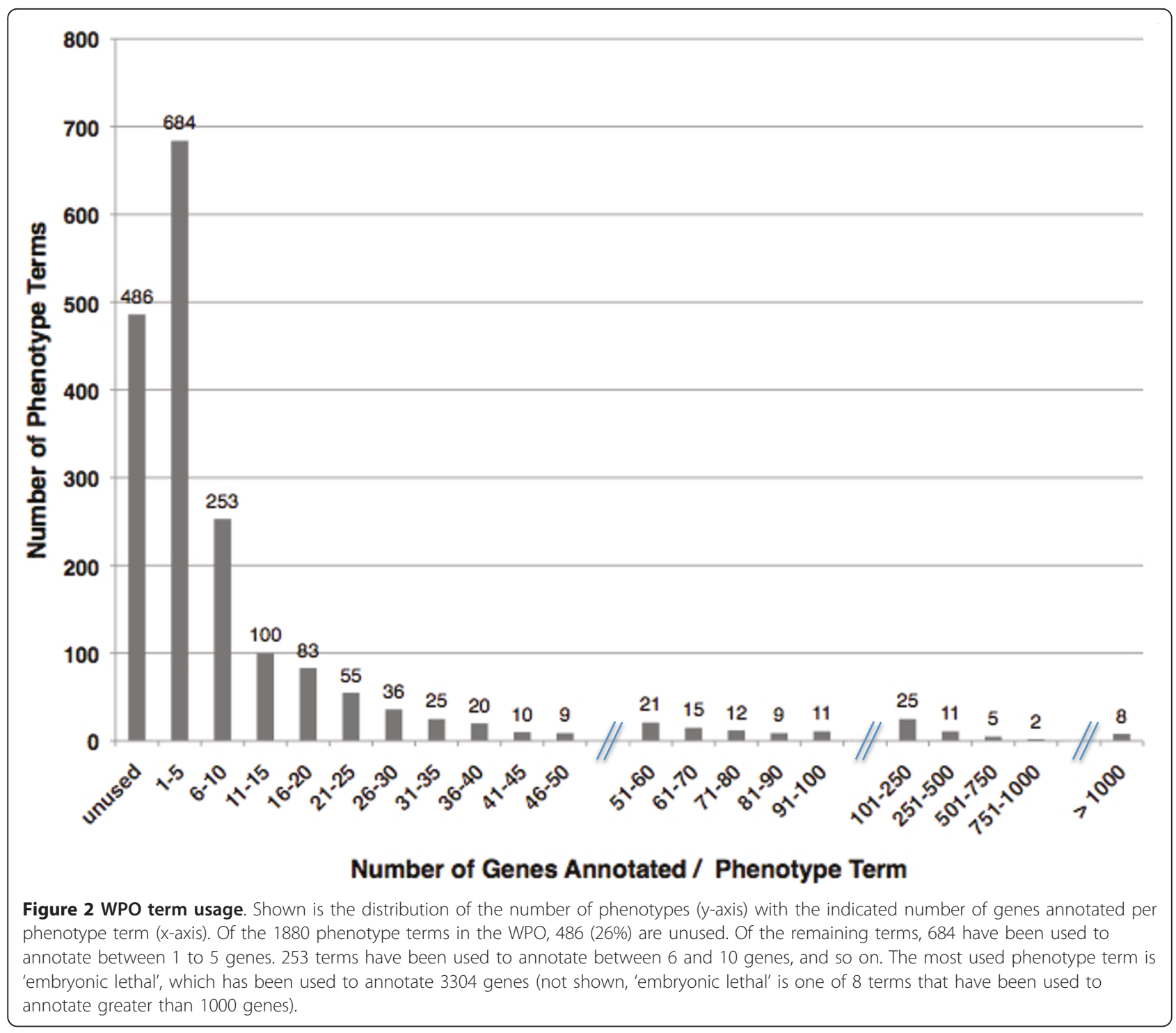

branch reflects our general policy of ontology development, which is to mirror the research direction of the community, and is influenced by the particular technological advantages offered by C. elegans as a model system, in this case RNAi.

\section{Expanding the scope and utility of the WPO}

Initially our ontology used 'Abnormal' as the root term, instead of 'Variant', and reflected the fact that "wild type" is defined as a genetically homogenous reference strain, Bristol $\mathrm{N} 2$, and for practical purposes, accepted as such by the C. elegans community. Recently, other nematodes besides C. elegans var. Bristol N2 have been incorporated into WormBase including five additional Caenorhabditis species (C. briggsae, C. remanei, C. brenneri, C. japonica and C. sp. 3 PS1010) and four non-Caenorhabditis nematodes (B. malayi, M. incognita, M. hapla and P. pacificus) [18].
However, the use of 'Abnormal' in WPO term names would preclude the annotation of phenotypes to these species. Moreover, the 'Abnormal' qualifier also prevents the annotation of closely-related C. elegans natural variants (e.g., C. elegans var. Hawaii) as characteristics vary among strains/ isolates of the same species, but are not deemed abnormal. An example of this is the deposition of a mating plug after copulation. C. elegans var. Bristol N2 does not deposit a mating plug after copulation, however C. elegans var. Hawaii does [28]. Therefore we chose to use 'Variant' as the root term to avoid comparing everything to Bristol N2.

In addition to replacing 'Abnormal' with 'Variant' as the root term, our phenotype ontology underwent a number of revisions with respect to term definitions so as to include non-hermaphroditic species. For example, the term 'egg morphology abnormal', was changed to 'egg morphology variant' and defined as, "Any variation 


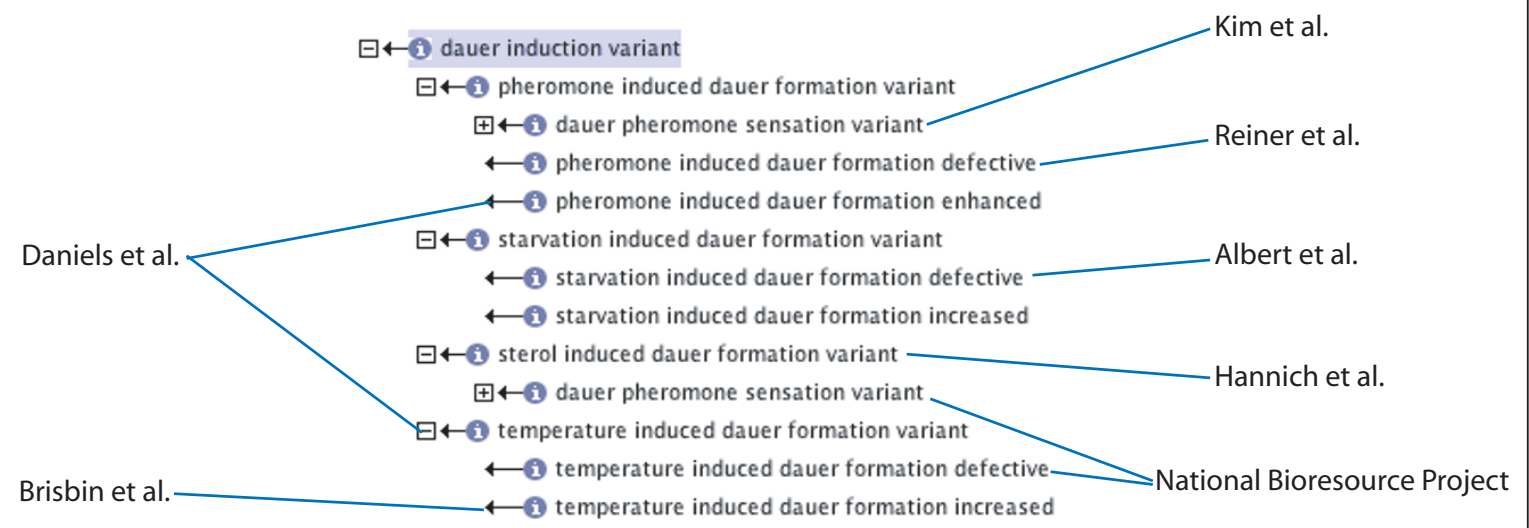

b)

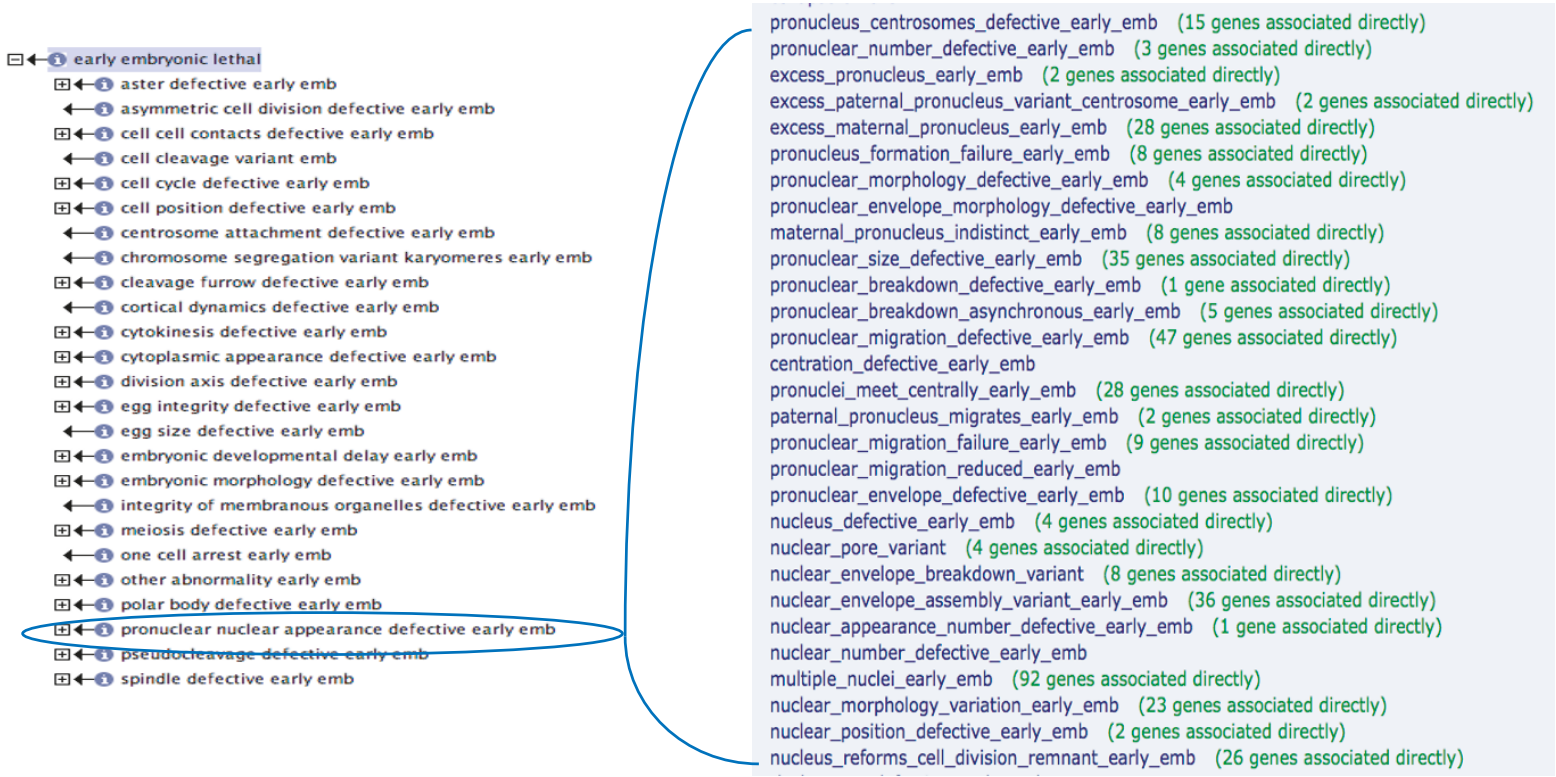

Figure 3 Forces driving the development of the WPO. Curation of C. elegans literature helps to increase the robustness of the phenotype ontology and we create terms as needed. Ontology views within OBO-Edit. (a) Blue lines point to the reference for the term. In some cases more than one term is created from a single reference [9,66-71]. (b) Expert input leads to extensive granularity in the ontology. There are 29 descendants of the 'pronuclear nuclear appearance defective early emb' branch (bracketed box), which was refined by soliciting feedback from the community.

in the overall structure or appearance of fertilized oocytes that are laid compared to those laid by control animals." Because we refer to control and not "wild type" or "N2", we are no longer limited to applying this term to a hermaphroditic species. Furthermore, the term can be used regardless of egg morphology differences among species, as the control is the reference strain. Phenotype terms still exist within the ontology to describe alterations in hermaphrodite-specific phenomena and new terms can be created to accommodate 
female and male specific terms from other nematode species. When we made this switch we retained the 'Abnormal' version of the term as a synonym so users accustomed to the previous nomenclature could still find terms of interest.

\section{Phenotype assignments to non- $C$. elegans nematodes}

As mentioned above, precluding the use of Bristol N2-specific phenotype terminology enables us to curate natural variants (e.g., Hawaii) and assign accurate and consistent phenotypes to non-Bristol N2 strains and mutants (Figure 4). The ability to query genomic databases via a standardized phenotype ontology used across species will facilitate the linking of evolutionary outcomes across those species with pertinent genetic changes, providing evolutionary biologists with a foundation for

\section{a)}

\section{C. briggsae Strain Report for AF16}

\begin{tabular}{|c|l|}
\hline Strain & AF16 \\
\hline Genotype & C. briggsae wild type. \\
\hline
\end{tabular}

The following phenotypes were observed in this experiment

\begin{tabular}{l|l}
\hline \hline \multicolumn{1}{c|}{ Phenotype } & \multicolumn{1}{c}{ Evidence } \\
\hline \hline biofilm absent head (Bah) & Darby C et al., 2007 Pubmed \\
\hline \hline carbon dioxide response variant & Hallem EA and Sternberg PW, 2008 \\
\hline \hline mate finding defective & Srinivasan J et al., 2008 Pubmed \\
\hline \hline
\end{tabular}

b)

\section{C. briggsae Variation Report for v53}

\begin{tabular}{c||ll}
\hline \hline $\begin{array}{c}\text { General } \\
\text { Information }\end{array}$ & $\begin{array}{l}\text { Variation: v53 } \\
\text { Variation type: allele }\end{array}$ & \\
& Affects Gene: & Cbr-she-1 \\
\hline
\end{tabular}

The following phenotypes were observed in this experiment

\begin{tabular}{|l||l}
\multicolumn{1}{|c||}{ Phenotype } & \multicolumn{1}{c}{ Reference(s) } \\
\hline \hline reduced brood size & Guo Y et al. \\
\hline $\begin{array}{l}\text { germ cell differentiation } \\
\text { variant }\end{array}$ & Guo Y et al. \\
\hline $\begin{array}{l}\text { hermaphrodite self } \\
\text { sterile }\end{array}$ & Guo Y et al. \\
\hline
\end{tabular}

Figure 4 C. briggsae phenotype assignments in WormBase C. briggsae is a nematode species that is closely related to C. elegans [72]. (a) Shown are excerpts from the AF16 strain page, a wild-type form of C. briggsae, which reports the associated phenotype annotations and the corresponding references that describe the controls for each of the experiments. (b) Shown are excerpts from the $v 53$ variation report page, listing observed phenotypes and corresponding references. v53 is a C. briggsae she-1 mutant. dissecting fundamental pathways and processes. For instance, even though both C. elegans and C. remanei descended from a gonochoristic ancestor, C. elegans exhibits hermaphroditism [29]. Recently, it has been argued that the evolution of hermaphroditism in Caenorhabditis can be attributed to a tra-2 mutation in the sexdetermination pathway that causes $X X$ spermatogenesis and a swm-1 mutation that allows these spermatids to self-activate [30]. Therefore, retrieving genes annotated to 'spermatogenesis variant' in these non-C. elegans species can provide a vocabulary useful to evolutionary biologists that choose to dissect these pathways.

\section{Annotating allele, RNAi and transgene overexpression phenotypes in C. elegans}

The utility of the Worm Phenotype Ontology is especially apparent when browsing Gene Summary pages, Variation Reports, Transgene Summary pages, and RNAi Reports at the WormBase site (http://www.wormbase.org/). The main portal for access to phenotypic data is through the WormBase Gene Summary page (Figure 5). The phenotype summary tables in the 'Function' section of the Gene Summary page includes a list of phenotype associations to a specific gene, as well as a list of phenotypes specifically reported as not being associated with that gene. Phenotypes not associated with a gene are prefaced by a 'Not' qualifier. This usage means researchers looked for this specific phenotype, but did not observe it in the reported experiment. For example, in Figure 5, the phenotypes 'methiothepin resistant', 'developmental delay' and 'locomotion variant' were not observed when the daf-2 gene was disrupted. The use of the 'Not' qualifier eliminates the need to duplicate every term in the WPO in the negative. A summary of the current nembers to allele, RNAi and transgene overexpression phenotypes are shown in Table 1.

\section{Data mining and searches}

WormBase has implemented a phenotype ontology search tool to integrate experimental phenotype data from RNAi experiments, alleles, and transgenes. Using the ontology search (found on the main WormBase page under searches) a user can input a term, phrase, synonym (e.g., Dpy) or ID to search for annotations connected to that term. For example if a user enters 'dumpy' in the search field (see Figure 6a) and selects the phenotype ontology, by default the search will look for 'dumpy' in the term name, definition and synonyms fields. The output (see Figure 6a) shows term names that contain 'dumpy' and terms that use 'dumpy' in their definitions. Each term shown in the browser is followed by a hypertext link listing the number of annotations in WormBase to each term and/or to children of that term (see Figure 6a). In addition, on the phenotype 


\section{daf-2 Phenotype Summary}

\begin{tabular}{|c|c|}
\hline Phenotype & Supporting Evidence \\
\hline sterile & Alleles:tm1236,sa223 \\
\hline locomotion_variant & Supported by 2 RNAi experiment(s) \\
\hline temperature_influenced_life_span_variant & Alleles:e1370 \\
\hline organ_senescence_variant & Alleles:e1370,e1368 \\
\hline small & Supported by 1 RNAi experiment(s) \\
\hline no_dauer_recovery & Alleles:sa231,sa230,sa223,m65 \\
\hline gene_expression_level_high & Alleles:e1370 \\
\hline life_span_variant & Supported by RNAi experiment(s) \\
\hline dauer_constitutive & $\begin{array}{l}\text { Alleles:sa193,e1370,sa231,sa229,sa197 } \\
\text { Supported by } 2 \text { RNAi experiment(s) }\end{array}$ \\
\hline pathogen_resistance_increased & Allele 1370. \\
\hline autophagic_cell_death_variant & Alleles: 1370 \\
\hline larval_lethal & Alleles:sa223 \\
\hline molt_defect & Supported by 1 RNAi experiment(s) \\
\hline lipid_composition_variant & Alleles:e1370 \\
\hline alae_secretion_variant & Supported by 1 RNAi experiment('s). \\
\hline embryonic_lethal & Supported by 1 RNAi experiment(s) \\
\hline male_tail_development_variant & Supported by 1 RNAi experiment(s) \\
\hline mianserin_resistant & Alleles:e1370 \\
\hline \multicolumn{2}{|c|}{ The following phenotypes have been reported as NOT observed in daf-2 } \\
\hline Phenotype & Supporting Evidence \\
\hline methiothepin_resistant & Nlleles:e1370 \\
\hline developmental_delay & upported by 1 RNAi experiment(s) \\
\hline locomotion variant & upported by 1 RNAi experiment(s) \\
\hline
\end{tabular}
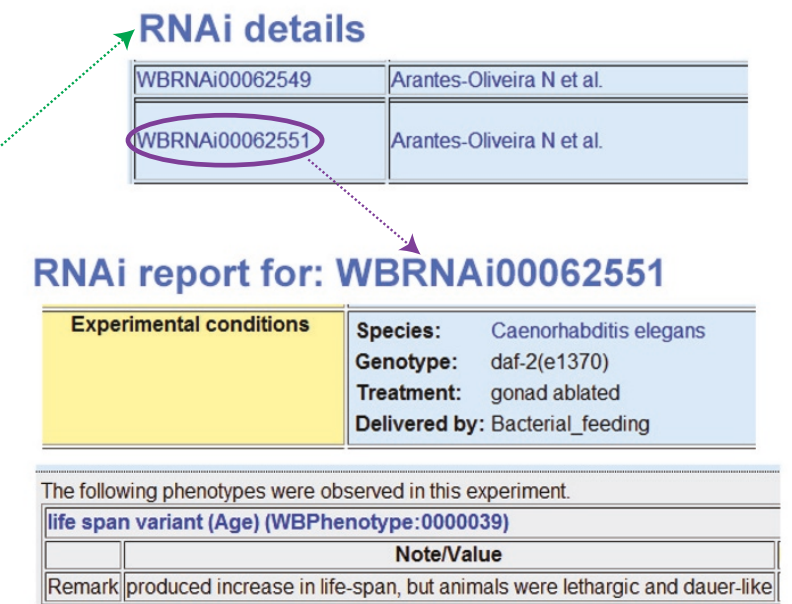

Remark produced increase in life-span, but animals were lethargic and dauer-like

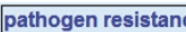
pathogen
increased

Figure 5 C. elegans phenotype assignments in WormBase. Shown are excerpts from the daf-2 gene page in WormBase. Phenotypes associated with alleles, RNAi experiments or transgenes (not shown) can be viewed in the phenotype summary tables. The e1370 allele object has its own specialized Variation Report' page that can be accessed through links, marked with a red oval, embedded in the phenotype summary tables on the Gene Summary page. The phenotype summary tables include a list of phenotypes associated with knockdown via RNAi for daf-2 (green oval). A more detailed overview of this RNAi experiment can be found within the 'RNAi details' section. The details section also contains links to a specific experiment, called the 'RNAi Report', via the WBRNAi ID (purple oval). The phenotype summary also includes 'Not' phenotype annotations (bottom left).

term pages in WormBase one can browse the term names in the ontology (see Figure 6b). Thus, when coupled with the new ontology search tool, the ontology's controlled vocabulary facilitates the retrieval of allele, RNAi, transgene, or strain objects that have equivalent phenotypes. In addition, the organization of the ontology facilitates the retrieval of objects annotated to phenotypes that are considered to be a more defined subclass of a phenotype term. For example one might want all the annotations to 'locomotion reduced', which would include 'fainter', 'paralyzed', etc., or one might just want the annotations that are directly associated with one of these terms (see Figure 1c).

In addition, to facilitate phenotype term enrichment analysis, the WPO contains a "WPO slim". A slim is a streamlined version of the ontology that contains a subset of the terms in the whole WPO. This subset is meant to give a broad overview of the ontology content without the details of the more specific granular terms.

\section{Using PATO-GO cross-products to integrate the WPO with} other organism databases

Phenotypes are typically described by using either a species-centric pre-composed ontology or using a more general post-compositional approach, drawing from various ontologies [31-35]. In a pre-composed ontology, phenotype terms are already defined and placed within the hierarchical structure of the ontology, such as the WPO. To describe a phenotype term using a post-compositional approach, a bipartite "EQ" (Entity + Quality) schema is employed and the entity of interest is described by a quality [36]. For example, in the case of the phenotype 'shrunken intestine', the entity is 'intestine' (WBbt:0005772) and the quality is 'shrunken' (PATO:0000585) [17]. The quality 
Table 1 Annotation summary ${ }^{\mathrm{a}}$

\begin{tabular}{lcc}
\hline Data Object & $\begin{array}{c}\text { Number of Genes } \\
\text { Annotated }\end{array}$ & $\begin{array}{c}\text { Number of Phenotype } \\
\text { Connections }^{\mathbf{b}}\end{array}$ \\
\hline Alleles & $4361^{\mathbf{c}}$ & 19824 \\
RNAi & $18834^{\mathrm{d}}$ & 297909 \\
Transgenes & $109^{\mathrm{e}}$ & 461 \\
(Overexpression) & & \\
\hline
\end{tabular}

${ }^{a}$ Data from the WormBase WS215 release.

${ }^{b}$ Includes both observed and 'Not' observed phenotypes. The number of phenotype connections is greater than the number of genes annotated, as each gene may have multiple phenotypes associated with them.

c Derived from 7066 alleles.

${ }^{d}$ Derived from 79068 RNAi experiments.

${ }^{\text {e }}$ Associated with 223 transgenes.

terms are derived from the Phenotype and Trait Ontology (PATO) [37], which can be used in conjunction with species-specific anatomical ontologies or cross-species entity ontologies [38,39]. This flexible post-compositional approach has already been employed in the annotation of human genotype-phenotype associations, as well as in model organism databases such as FlyBase (Drosophila) and ZFIN (zebrafish) [31,34].

We used a pre-composed approach to create the WPO because pre-composed ontologies are able to incorporate community specific jargon, some of which is not amenable to the EQ schema (because it fails to capture the term's biological complexity). The 'kinker' phenotype is one case in which non-C. elegans users would not intuitively grasp its relationship to locomotory behavior without the benefit of a pre-coordinated hierarchy. Although our pre-composed ontology serves the data mining needs of nematode researchers, its lack of crossoperability would have the effect of rendering worm phenotype terms as opaque and less accessible to researchers using other organisms in their research.

To promote interoperability across different MODs and other biological databases we generated logical equivalence relationships (or cross-products) between process-oriented phenotype terms in the WPO and PATO-based EQ descriptions [17]. The availability of cross-products means that phenotype annotations to the pre-composed terms can be automatically converted to their corresponding EQ descriptions and vice versa. This conversion will facilitate communication between diverse organism phenotype ontologies and potentially improve data integration across communities (Figure 7a). We chose to generate equivalence mappings to the process-oriented phenotype terms because of their partial overlap with the Gene Ontology (GO), whose widespread adoption by distinct groups has played a crucial role in the integration of biological data [16]. Currently, the equivalence mappings we generated are not integrated into WormBase, but they can be accessed and viewed with an ontology editor (OBO-Edit, see Methods).

\section{Benefits of WPO-GO cross-products}

The cross-products were generated manually so as to ensure logical coherence across ontologies and confirm biological validity. For instance the WormBase, FlyBase (http://flybase.org) and Mouse Genome Informatics (http://www.informatics.jax.org) databases contain 585, 99, and 945 'cell death' phenotype annotations, respectively (Figure 7b). Some of these phenotype associations appear to be conserved between their vertebrate and invertebrate gene orthologs, which suggests that mining genotype-phenotype connections from different organisms can lead to potentially useful predictions in other complex biological systems. In addition to constructing basic equivalence relations (based on semantic similarity), we were occasionally able to generate non-obvious yet biologically relevant Worm PO-GO cross-products by scrutinizing the literature and/or the definitions and synonyms of both WPO and GO-process terms, such as 'quiescence variant' and 'sleep' (Additional file 1) [40].

Another significant benefit of such equivalence mappings is the unmasking of cryptic terminologies that are often embedded within many individual phenotype terms or incorporated as synonyms. In other words, nematode 'species-centric' jargon (for instance, 'nose touch defective') becomes transparent to an outside researcher since the accurate equivalence mapping made to the parent term (namely, 'mechanosensation variant') would apply to the descendants as well (Additional file 2). This is a consequence of the is_a relationship, the more granular term (descendent) has general properties that it has inherited from its parent term [41].

Therefore the WPO, in conjunction with its validated equivalence mappings, can be utilized for cross-species queries and analyses of phenotype data derived from diverse resources. Although the simple pair-wise strategy can give incomplete results for complex phenotypes involving classes from more than one other ontology, this marks a good start towards constructing equivalences mappings in the WPO.

\section{Community driven evolution of the WPO}

Our understanding of biology evolves over time. The underlying goal of community engagement is to respond to and provide the necessary channels to accommodate changes, while maintaining coherence and best practice methods during ontology development [42]. With this goal in mind, we have provided a means for user participation in the development and maintenance of the WPO.

Users can directly interact with phenotype curators through the online allele submission form (which can be accessed at http://tazendra.caltech.edu/ azurebrd/cgibin/forms/allele.cgi, See Figure 8). The submission form gives users the option to browse the current ontology using term names, synonyms, or phenotype IDs (e.g. 
a)

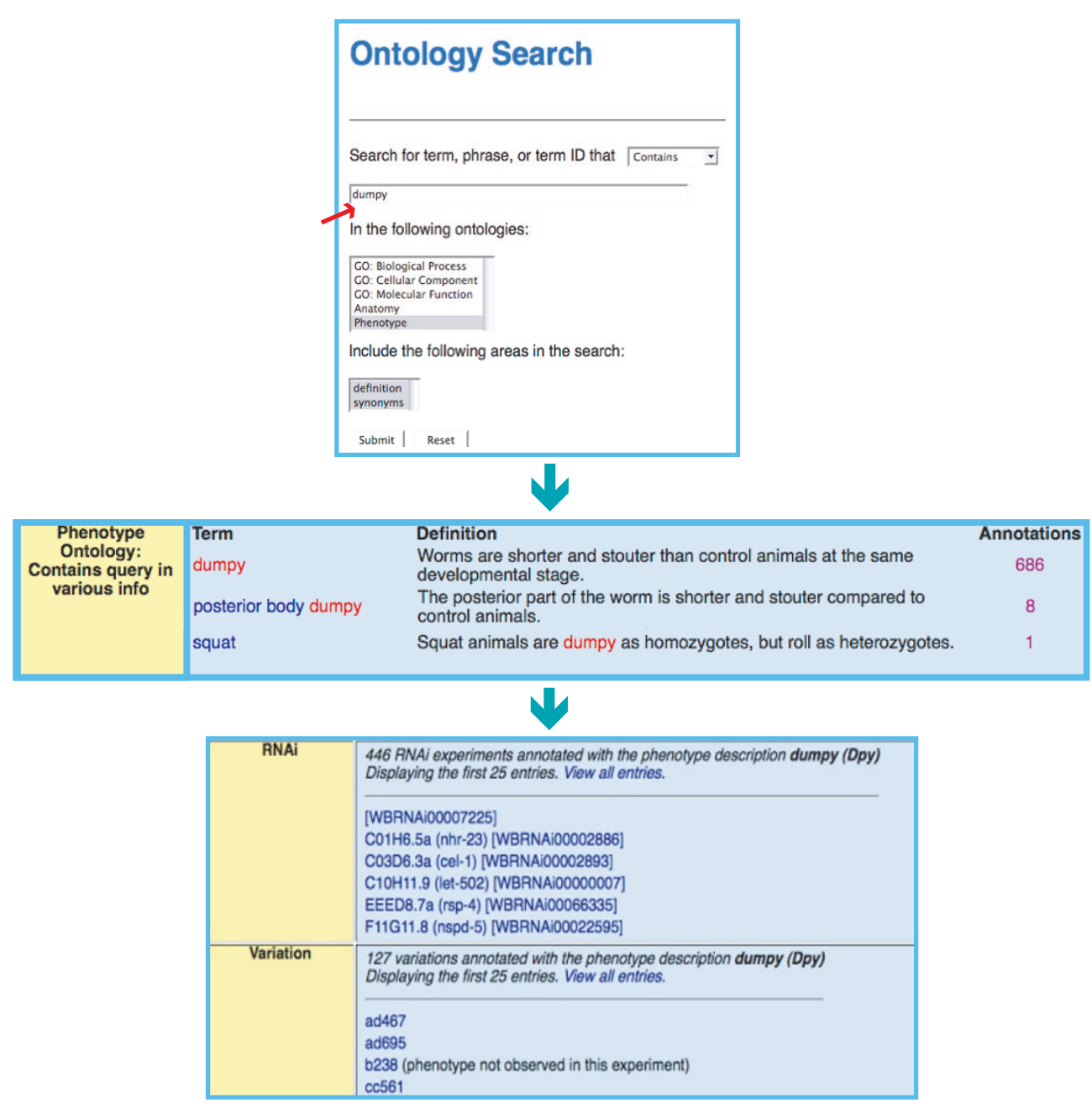

b)

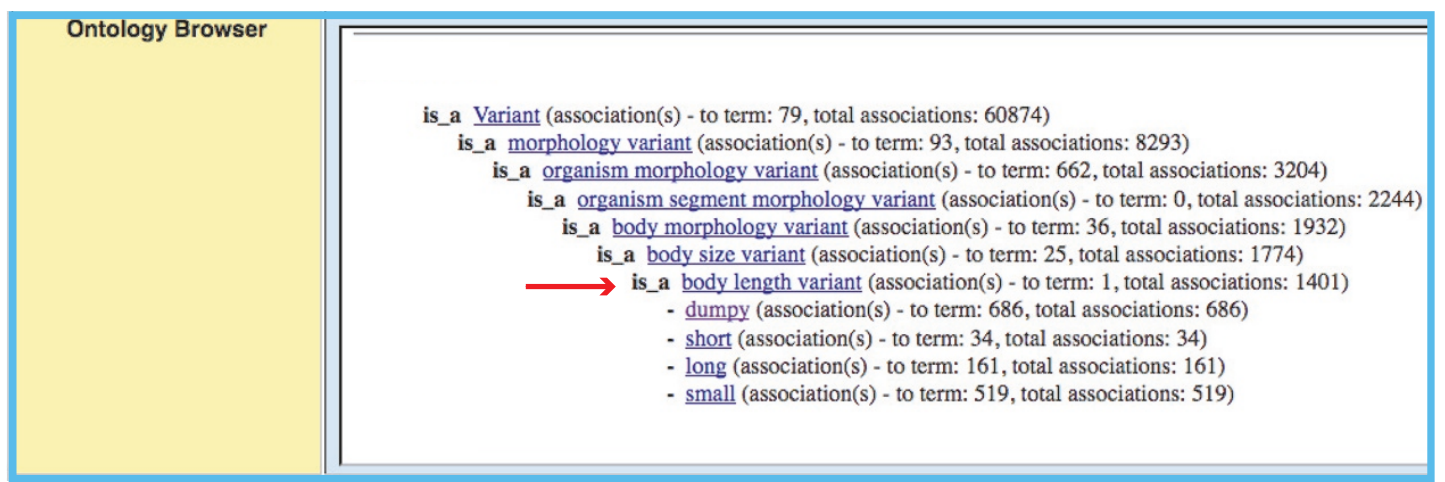

Figure 6 Data mining using the ontology search tool in WormBase. (a) A user may enter a query term in the search box; in this case 'dumpy' is used as an example. Results displayed in the output include the terms that contain 'dumpy' within the term name or within its definition (highlighted in red). Clicking on the number to the right, which indicates the total number of annotations to each term, retrieves RNAi, allele (variation) and transgene objects associated with a phenotype. Displayed is a portion of the 686 annotations made to 'dumpy'. There are RNAi and variation objects associated with this term, but no transgene data. (b) Included in the ontology search output (shown here for 'dumpy') is a window that allows the user to browse the ontology. If a user clicks on a term, the children of that term are revealed as well as the number of genes associated to that term. Shown is one gene directly annotated to 'body length variant' (red arrow), but 1401 total associations are indicated, as this number includes all the annotations to the children ('dumpy', 'short', 'long' and 'small'). 


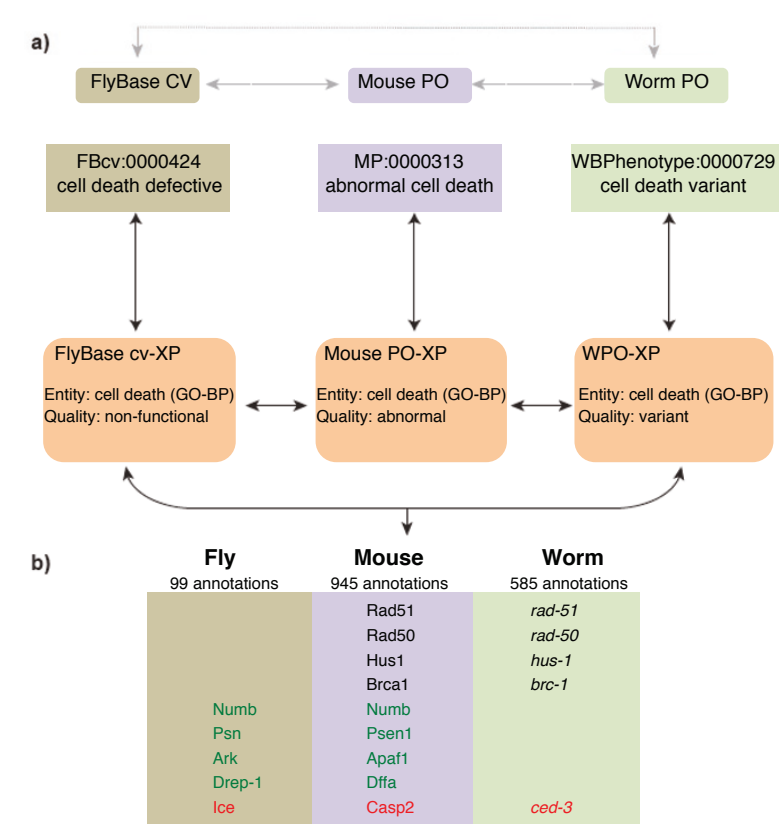

Figure 7 Integrating phenotype ontologies across evolutionarily divergent species. (a) Conceptual diagram depicting how multiple orthogonal phenotype ontologies (FlyBase Controlled Vocabulary, Mouse Phenotype Ontology, Worm Phenotype Ontology) can interact with each other via equivalence relationships (cross-products indicated by orange boxes). The example used here pertains to the 'cell death' process. XP stands for 'cross-product' and GO-BP stands for 'Gene Ontology Biological Process' (b) The table displays some of the phenotype annotations to genes relating to cell death anomalies in fly (Drosophila melanogaster), mouse (Mus musculus) and worm (Caenorhabditis elegans). Annotations were retrieved directly from their respective model organism databases (FlyBase, MGI, WormBase). Red font indicates conserved genes among all the depicted species. Green font shows conserved genes between D. melanogaster and $M$. musculus. Black font shows conserved genes between C. elegans and M. musculus.

dumpy, Dpy, WBPhenotype:0000583). Users can also propose new phenotype terms or suggest revisions to the subject matter or placement of existing terms within the ontological tree. Such critical evaluation would ensure that the WPO would evolve alongside the nematode research we are committed to archiving.

\section{Discussion}

We have developed the Worm Phenotype Ontology (WPO), a standardized syntax to classify and organize phenotype descriptions for C. elegans and other nematodes. The WPO will lead to consistency in phenotype description and an increase in curatorial efficiency at WormBase. The benefits of a controlled and structured phenotype vocabulary extends beyond WormBase users and will help integrate data from many different sources into a common body of knowledge, and facilitate data mining and comparisons across species. Hence, the WPO functions as a knowledge-based resource not only for $C$. elegans biologists, but also the larger biomedical, nematology, and bioinformatics communities.

\section{Improving data mining of phenotypic information}

Search tools play an important role in the retrieval and display of bioinformatics data [43]. As stated above, WormBase has an ontology search that allows users to retrieve annotations to specific genes based on certain criteria, such as phenotypes. In addition, WormMart (http://wiki.wormbase.org/index.php/Data_mining: WormMart) serves as a more general data mining tool. We hope to extend the functionality of other existing WormBase tools to help in the dissemination of phenotype-based information, such as viewing phenotype annotations within the Genome Browser [44], which could potentially expedite positional cloning and gene mapping. Such a feature for the mouse genome has already been implemented by MGI [14]. An additional enhancement would be the incorporation of images and/or movies that represent a phenotype class.

In the future, WormBase also plans to implement the ability to conduct searches for allele, RNAi, transgene, or strain objects that do/do not exhibit a combination of defined phenotypes. This capability will allow users to further differentiate between allele phenotype classes. For example, one could distinguish between genes that perform a general excitatory role in neurotransmission versus genes that function only in specific behaviors/cell types. This capability will also be extended to performing combinatorial searches across ontologies (e.g., the anatomy ontology) in order to define genes that have been shown to be expressed, or to act, in specified cell types.

Given the expected increase of "other nematode" phenotypes in WormBase, we hope to provide search tools that render complex evolutionary questions amenable to computational analyses (Additional file 3). One example would be the ability to identify genes involved in the genetic pathways associated with hermaphroditic reproduction in different species that have undergone convergent evolution, as hermaphroditism has occurred independently in several clades during recent nematode evolution $[45,46]$. The basis for this type of analysis would be to examine phenotypes that are specifically associated with hermaphroditism. For example, C. elegans hermaphrodites require $f \circ g-2$ to regulate the onset of spermatogenesis $[47,48]$. In contrast, the related species C. briggsae lacks fog-2 [49], but requires she-1 for execution of spermatogenesis in hermaphrodites [50]. A query for the 'spermatogenesis defective hermaphrodite' term would allow users to make the inference that both fog- 2 and she- 1 may act in the same pathway (Additional file 3). Additionally, such search tools would permit the 


\section{http://tazendra.caltech.edu/ azurebrd/cgi-bin/forms/allele.cgi}
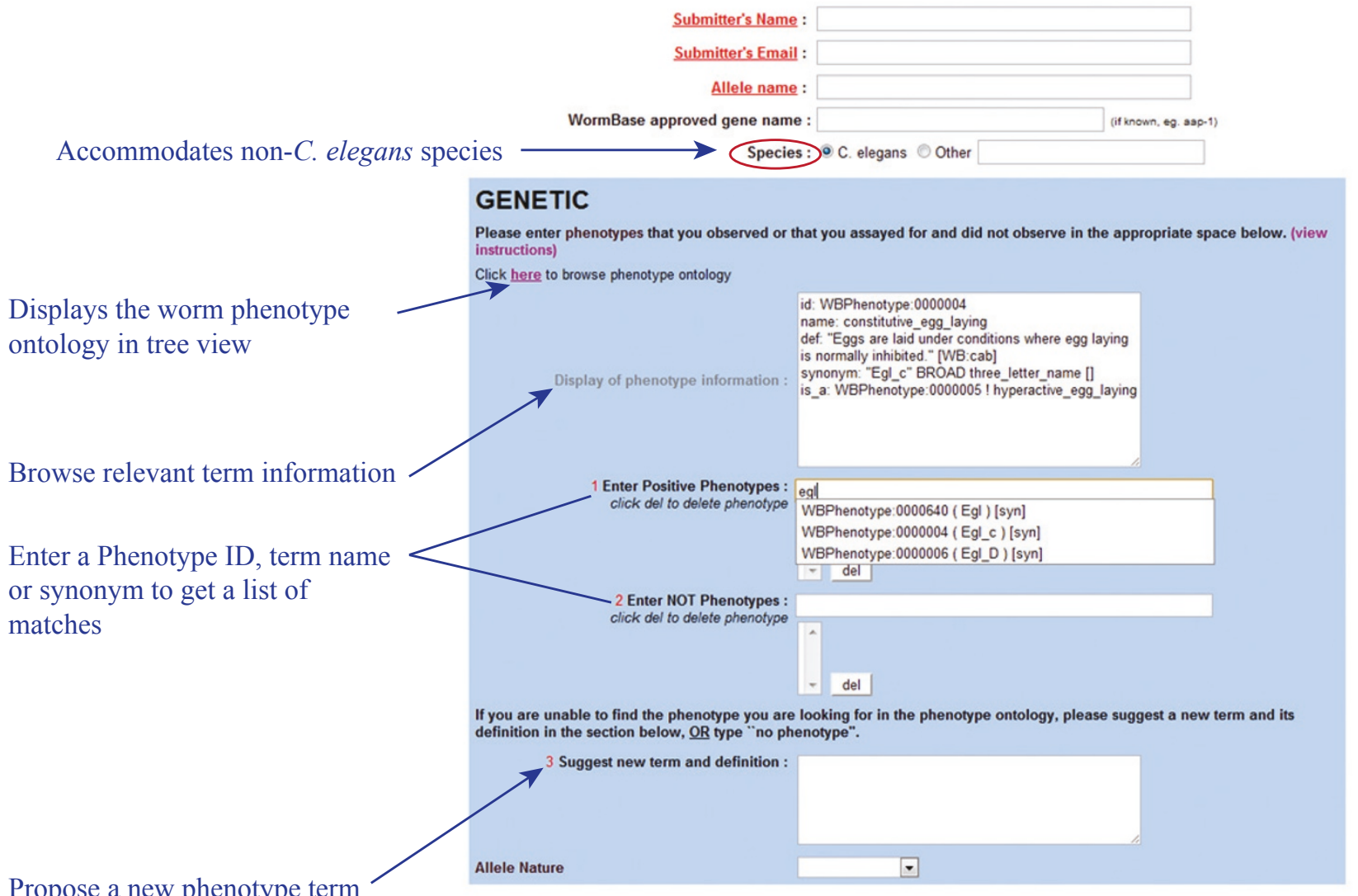

Propose a new phenotype term

Allele Nature

Figure 8 Online user submission form for alleles. The form allows users to browse the Worm Phenotype Ontology, assign phenotype terms to alleles or propose changes to the existing phenotype ontology. Submissions are reviewed prior to entry into the database. This form can also be accessed from the Allele data link on the WormBase Online Data Submission forms page at http://www.wormbase.org/db/curate/

online_forms.

identification of gene products that are co-opted for divergent functions across species. For instance, gld-1 promotes spermatogenesis in C. elegans hermaphrodites [51], whereas C. briggsae gld-1 appears to play the opposite role in germline sex determination (oogenesis) [49].

One strategy to quickly increase these cross-species data in WormBase is to curate RNAi experiments conducted with other nematodes such as Heterorhabditis bacteriophora [52]. The infrastructure for RNAi curation of other species exists at WormBase, the only caveat being that a stable reference genome is necessary to attach the sequence used in an RNAi experiment to a specific gene. In addition, we will rely on community input on mutant phenotypes in other species to help spearhead this effort.

Another opportunity for improving data synthesis is to improve the ability to store and retrieve phenotype information for syndromes in C. elegans. For example,
'Lag' (lin-12 and $g l p-1)$ phenotypes consist of 'nose twisted', 'no rectum', and 'excretory system development variant' phenotypes, a specific phenotype combination assigned to genes that are shared in processes controlled by the lin-12 and glp-1 Notch receptors in C. elegans [53]. One possibility is to develop a syndrome database model that captures the phenotype classes associated with each syndrome object. In the 'Lag' case mentioned above, we would create a 'Lag' syndrome object and if an allele, for example, displayed the 'nose twisted', 'no rectum', and 'excretory system development variant' phenotypes, it would automatically be associated with the 'Lag' syndrome. An extension of this approach, already being implemented by the Human Phenotype Ontology (HPO) group [54], would be to also create a search tool that looks not only at exact phenotype term matches for a specific syndrome, but is also able to traverse the pre-coordinated ontological structure for 
related terms and assign probability scores (significance threshold) for such results. For example, if an animal displays 'no rectum', 'excretory system development variant' and 'nose morphology variant' (the parent of 'nose twisted'), it would receive a lower but potentially significant confidence score for the 'Lag' syndrome because it does not contain the exact 'nose twisted' term, but has an exact match for the other two phenotypes.

\section{Intersections between orthogonal WPO, GO, and other ontologies}

It is important to note the differences between annotation using the WPO compared to the GO. Whereas phenotype annotations are inextricably linked to the genetic background and conditions used to assay the phenotype, and these conditions are generally incorporated in the term's name or definition, manually assigned GO biological process annotations based upon mutant phenotypes (Inferred from Mutant Phenotype, or IMP) are meant to capture, as closely as possible, the biological processes directly affected by the gene. Thus, to assess an appropriate GO biological process annotation for a gene based upon a mutant phenotype, curators may consider additional information, such as the molecular identity of the gene product or, if known, the point in the process at which the gene product is believed to act. For example, a bHLH transcription factor that controls transcription of genes involved in muscle cell differentiation may exhibit a mutant phenotype of altered muscle contraction similar to that of mutations in genes encoding sarcomeric proteins. However, whereas both genes could be annotated to a 'muscle contraction variant' phenotype, the latter could be accurately annotated to the GO biological process term muscle contraction, while the former more accurately annotated to a term that reflects its role in regulation of muscle-specific gene transcription. The WPO and GO annotations would intersect at the level of a musclerelated process, but would differ in that the GO biological process annotation would capture the more specific roles of the individual gene products.

Logical equivalence relationships between ontologies also need to account for dependencies such as genetic background or assay and environmental conditions, and cross-species comparisons will be more meaningful when cross-products also incorporate this information in a standardized way using the various ontologies currently in development (OBI, the ontology for biomedical investigations; ChEBI, the chemical information ontology [55]; FIX, the ontology of physico-chemical methods and properties (http://www.obofoundry.org), etc.). Additional insight might be gained by these mappings and they will complement the synthesis of information that represents a $\mathrm{GO}$ annotation.
Possible solutions to/challenges in creating crossproducts to anatomy-based phenotypes

Much like the GO, the Worm Phenotype Ontology is continually undergoing active development. In addition to maintaining the existing ontological framework and enriching it with new phenotype classes, we are now in the process of generating cross-product ontologies for the non-process oriented phenotype classes (anatomy-based, chemical-based etc.). Besides facilitating phenotype comparisons across species, such equivalence relationships will help to build and maintain the WPO itself. As mentioned earlier, cross-product descriptions must be unambiguous and biologically sound in order to efficiently integrate phenotype data across various research organisms. However, the task of creating new cross-product ontologies, for example anatomy-based phenotypes, is accompanied by its own set of challenges.

For example, if we rely exclusively on the worm anatomy ontology developed in-house (http://www.obofoundry.org/cgi-bin/detail.cgi?id=worm_anatomy) for generating cross-products to anatomy-based phenotype terms, the utility of such equivalence mappings could potentially be restricted to the $C$. elegans community. One possible solution is to include UBERON [56], a uniform multi-species anatomy ontology, while generating cross-products to phenotypes involving certain generic anatomical entities (such as intestinal cell, striated muscle etc). UBERON has the added benefit of containing links to over 9,300 classes in other species-centric anatomical ontologies (besides the worm anatomy ontology), which could potentially simplify the retrieval of anatomy-based phenotypes across species. This is at best a partial solution because currently there is limited sharing of anatomical entities between UBERON and the worm anatomy ontology (40/6207 classes).

\section{Prediction of gene function and human disease models}

Computational analyses of phenotype ontologies promotes the discovery of similarities between related phenotype abnormalities, which can subsequently be used for clinical diagnostic queries or as a basis for integrating phenotype and gene expression data sets to predict gene function $[57,58]$, or other phenomena associated with complex human diseases.

The Worm PO-GO cross-product ontology (see above) is a valuable tool in terms of unmasking genes involved in fundamental processes that are shared among different species such as cell death, cell cycle etc. However, these equivalence relationships might not be as insightful when it comes to dissecting 'orthologous phenotypes' or phenotypes that arise from the disruption of a set of evolutionarily conserved genes that are differentially manifested across species. A recent study reported a method for identifying non-obvious equivalences between 'orthologous 
phenotypes' (phenologs) and human disease models [59]. These findings could be enriched to make predictions about gene networks involving phenologs by exploiting the hierarchical structure of multiple pre-coordinated phenotype ontologies. Namely, this would involve the recovery of genes that are annotated to phenotypes considered to be of a more specific subclass of the phenolog of interest, thereby aiding in the identification of additional candidate disease genes (Additional file 4). These results depend on the assumption that there can be a 1:1 equivalence mapping between the different phenotype classes of evolutionary distant species, for example human retinoblastoma eye cancer and ectopic vulvae in C. elegans [60,61].

At present, there is no individual platform that has the capacity to retrieve all existing mammalian and nonmammalian phenotypes that mirror well-documented human disorders, such as neurofibromatosis or Marfan syndrome. One plausible strategy would be to push towards more disease annotation; for instance, linking a specific mutation (and consequently its annotated phenotypes) to a human disease entry in OMIM [62]. This would presumably lead to the identification of other potential 'phenologs' thereby providing insights into evolutionary developmental biology and human disease states.

\section{Conclusions}

Now that a framework for the Worm Phenotype Ontology is in place, further development and refinement of the ontology will occur in parallel with phenotype annotation; thus, evolution of the ontology will reflect the developing complexity with which phenotypes are described in nematodes. In addition to catering to the data mining needs of nematode biologists, one of our objectives is to make worm phenotypes accessible to the entire research community. In collaboration with other databases we ultimately envision the development of a web-based platform that integrates phenotype data, and data synthesis, across all MODs and other biological databases.

\section{Methods}

We use OBO-Edit, under active development by the Gene Ontology Consortium (GOC) [63], for ontology development, refinement, and expansion. Ontology updates are committed to the WPO using CVS (Concurrent Versions System), served from a local PostgreSQL database, so that multiple curators can access and edit the ontology simultaneously. To facilitate internal collaboration on the development of the WPO, we have set up an internal web-based tool so that curators working on different phenotype-based data types (i.e., RNAi, allele, cell function, etc.) can request new terms. Along with the suggested term, curators suggest a definition and hierarchical placement within the ontology. The WormBase community can also request phenotype terms via an allele submission form (see Figure 8). A current version of the WPO is also available to the public at The OBO Foundry (Open Biological and Biomedical Ontologies) [64], which can be accessed here: http:// caltech.wormbase.org/cvsweb/PhenOnt/ or from http:// www.obofoundry.org/cgi-bin/detail.cgi?

id=worm_phenotype.

OBO-Edit was also used to generate equivalence mappings for process-oriented phenotypes using the methodology described by Mungall and colleagues [17]. Each phenotype description consists of the following elements: E, the type of Gene Ontology (GO) process entity that is affected; $Q$, the quality borne by the entity. We refer to this collection of equivalence mappings as the Worm PO-GO-XP ontology (where XP stands for cross-product), which can be accessed at http://caltech. wormbase.org/cvsweb/PhenOnt/.

Allele and transgene phenotypes were initially curated via Phenote (http://www.phenote.org), a software application that facilitates phenotype annotation using ontologies. As of October 2009, we switched to a web-based ontology annotation tool (developed in-house; details to be described elsewhere) for the curation of alleles, transgenes and strains. RNAi sequence mapping tools were developed in-house.

\section{Additional material}

\begin{abstract}
Additional file 1: Figure S1. Construction of non-obvious yet biologically relevant equivalence mappings. (a) Equivalence

relationship between the 'quiescence variant' phenotype class and its corresponding EQ description. $\mathrm{E}=\mathrm{GO}$ term 'sleep' and $\mathrm{Q}=\mathrm{PATO}$ term 'variant'. (b) This table displays some of the phenotype annotations to genes relating to sleep anomalies in fly (Drosophila melanogaster), mouse (Mus musculus) and worm (Caenorhabditis elegans). Annotations were retrieved directly from their respective model organism databases (FlyBase, MGl, WormBase). Red font indicates conserved genes among all the depicted species. Green font shows conserved genes between $D$. melanogaster and C. elegans. Black font shows conserved genes between
\end{abstract} D. melanogaster and M. musculus.

Additional file 2: Figure S2. Cross-products assigned to the 'mechanosensation variant' class apply to all of the granular subclasses as well (such as 'nose touch defective'). Shown on left is the 'mechanosensation variant' term in the context of the WPO. Its crossproducts are indicated by dashed lines in OBO-Edit on the Cross Products Table Here 'Intersection Genus' represents 'Quality' and the 'Discriminating Relationships' represent 'Entity'. Also shown (blue arrow) is the term definition of one of the subclasses ('nose touch defective'). The cross product to the parent applies to this child term as well.

Additional file 3: Figure S3. Potential application of mining phenotypic data for multiple nematode species. Shown is a theoretical table generated by querying WormBase for 'spermatogenesis defective hermaphrodite'. The results include genes (annotated to this phenotype term) along with their corresponding species and reference. Green font depicts genes with different molecular functions that are both involved in tra-2 repression to promote XX spermatogenesis (convergence) and red font depicts a gene that has been co-opted for an alternate function in C. briggsae.

Additional file 4: Figure S4. Exploiting the hierarchy of precoordinated phenotype ontologies to acquire data on gene networks involving 'orthologous phenotypes' and their relationship 
to human disease. The example illustrated is the Notch/Delta family (pink oval), its connection to human disease (green oval) and a corresponding phenotype connection to the mouse and worm phenotype ontologies ('abnormal hematopoiesis' and 'germline proliferation variant' are the respective terms). Red font points to direct associations with the parent terms and blue fonts bracket the connections to the descendent terms.

\section{Acknowledgements}

We thank Raymond Lee and Christian Grove for helpful discussions and advice; Igor Antoshechkin for assistance with RNAi phenotype curation tools and advice; Juancarlos Chan for assistance with curation tools for alleles and transgene overexpression; Chris Mungall and Amina Abdullah for the impetus to include equivalences for the WPO; Kris Gunsalus for input on early embryonic lethal phenotype suggestions; Norie de la Cruz for his work on the phenotype ontology search tool and fellow curators at WormBase for their valuable input. We also thank Christian Grove, Ranjana Kishore, Raymond Lee, Chris Mungall, Kimberly Van Auken, Cheryl Van Buskirk, and Xiaodong Wang for comments on the manuscript. Supported by U.S.P.H.S grant P41HG0223 to PWS, an investigator of the Howard Hughes Medical Institute.

\section{Author details}

${ }^{1}$ Howard Hughes Medical Institute, California Institute of Technology, Pasadena, CA 91125, USA. 'Division of Biology, California Institute of Technology, Pasadena, CA 91125, USA.

\section{Authors' contributions}

$C A B$ and PWS initiated this project. CAB, GS, JSF and KY further developed the WPO, namely creating, defining and placing terms in the ontology. JSF and GS generated and maintained the WPO-GO XP ontology. GS, JSF and $C A B$ wrote the paper with valuable discussions and critical contributions at all stages of the project from KY and PWS. All authors read and approved the final manuscript.

Received: 12 August 2010 Accepted: 24 January 2011

Published: 24 January 2011

\section{References}

1. Brenner S: The genetics of Caenorhabditis elegans. Genetics 1974, 77(1):71-94.

2. Lewis EB: Genetic control and regulation of developmental pathways. New York: Academic Press; 1964.

3. Muller HJ: Further Studies on the Nature and Causes of Gene Mutations. Proceedings of the 6th International Congress of Genetics 1932, 213-255.

4. Consortium: Genome sequence of the nematode $C$. elegans: a platform for investigating biology. Science 1998, 282(5396):2012-2018.

5. Hillier LW, Coulson A, Murray Jl, Bao Z, Sulston JE, Waterston RH: Genomics in C. elegans: so many genes, such a little worm. Genome Res 2005, 15(12):1651-1660.

6. Fire A, Xu S, Montgomery MK, Kostas SA, Driver SE, Mello CC: Potent and specific genetic interference by double-stranded RNA in Caenorhabditis elegans. Nature 1998, 391(6669):806-811.

7. Bargmann Cl: High-throughput reverse genetics: RNAi screens in Caenorhabditis elegans. Genome Biol 2001, 2(2), REVIEWS1005.

8. Moerman DG, Barstead RJ: Towards a mutation in every gene in Caenorhabditis elegans. Brief Funct Genomic Proteomic 2008, 7(3):195-204.

9. Yamazaki $Y$, Akashi R, Banno $Y$, Endo T, Ezura H, Fukami-Kobayashi K, Inaba K, Isa T, Kamei K, Kasai F, Kobayashi M, Kurata N, Kusaba M, Matuzawa T, Mitani S, Nakamura T, Nakamura Y, Nakatsuji N, Naruse K, Niki H, Nitasaka E, Obata Y, Okamoto H, Okuma M, Sato K, Serikawa T, Shiroishi T, Sugawara H, Urushibara H, Yamamoto M, Yaoita Y, Yoshiki A, Kohara Y: NBRP databases: databases of biological resources in Japan. Nucleic Acids Res 2010, 38 Database: D26-32.

10. Schwarz EM: Genomic classification of protein-coding gene families. WormBook 2005, 1-23.

11. Huang LS, Sternberg PW: Genetic dissection of developmental pathways. WormBook 2006, 1-19.
12. Costanzo MC, Skrzypek MS, Nash R, Wong E, Binkley G, Engel SR, Hitz B, Hong EL, Cherry JM: New mutant phenotype data curation system in the Saccharomyces Genome Database. Database Oxford; 2009, 2009 bap001.

13. Jaiswal P, Ware D, Ni J, Chang K, Zhao W, Schmidt S, Pan X, Clark K, Teytelman L, Cartinhour S, Stein L, McCouch S: Gramene: development and integration of trait and gene ontologies for rice. Comp Funct Genomics 2002, 3(2):132-136.

14. Smith $C L$, Eppig JT: The Mammalian Phenotype Ontology: enabling robust annotation and comparative analysis. Wiley Interdiscip Rev Syst Biol Med 2009, 1(3):390-399.

15. Bard JB, Rhee SY: Ontologies in biology: design, applications and future challenges. Nat Rev Genet 2004, 5(3):213-222.

16. Ashburner M, Ball CA, Blake JA, Botstein D, Butler $H$, Cherry JM, Davis AP, Dolinski K, Dwight SS, Eppig JT, Harris MA, Hill DP, Issel-Tarver L, Kasarskis A, Lewis S, Matese JC, Richardson JE, Ringwald M, Rubin GM, Sherlock G: Gene ontology: tool for the unification of biology. The Gene Ontology Consortium. Nat Genet 2000, 25(1):25-29.

17. Mungall CJ, Gkoutos GV, Smith CL, Haendel MA, Lewis SE, Ashburner M: Integrating phenotype ontologies across multiple species. Genome Biol 2010, 11(1):R2.

18. Harris TW, Antoshechkin I, Bieri T, Blasiar D, Chan J, Chen WJ, De La Cruz N, Davis P, Duesbury M, Fang R, Fernandes J, Han M, Kishore R, Lee R, Muller HM, Nakamura C, Ozersky P, Petcherski A, Rangarajan A, Rogers A, Schindelman G, Schwarz EM, Tuli MA, Van Auken K, Wang D, Wang X, Williams G, Yook K, Durbin R, Stein LD, Spieth J, Sternberg PW: WormBase: a comprehensive resource for nematode research. Nucleic Acids Res 2010, , 38 Database: D463-467.

19. Smith B: The logic of biological classification and the foundations of biomedical ontology. London: King's College Publications; 2005.

20. Stark C, Breitkreutz BJ, Chatr-Aryamontri A, Boucher L, Oughtred R, Livstone MS, Nixon J, Van Auken K, Wang X, Shi X, Reguly T, Rust JM, Winter A, Dolinski K, Tyers M: The BioGRID Interaction Database: 2011 update. Nucleic Acids Res 39:D698-704.

21. Muller HM, Kenny EE, Sternberg PW: Textpresso: an ontology-based information retrieval and extraction system for biological literature. PLOS Biol 2004, 2(11):e309.

22. Girard LR, Fiedler TJ, Harris TW, Carvalho F, Antoshechkin I, Han M, Sternberg PW, Stein LD, Chalfie M: WormBook: the online review of Caenorhabditis elegans biology. Nucleic Acids Res 2007, , 35 Database: D472-475.

23. Gonczy P, Echeverri C, Oegema K, Coulson A, Jones SJ, Copley RR, Duperon J, Oegema J, Brehm M, Cassin E, Hannak E, Kirkham M, Pichler S, Flohrs K, Goessen A, Leidel S, Alleaume AM, Martin C, Ozlu N, Bork P, Hyman AA: Functional genomic analysis of cell division in C. elegans using RNAi of genes on chromosome III. Nature 2000, 408(6810):331-336.

24. Kamath RS, Fraser AG, Dong Y, Poulin G, Durbin R, Gotta M, Kanapin A, Le Bot N, Moreno S, Sohrmann M, Welchman DP, Zipperlen P, Ahringer J: Systematic functional analysis of the Caenorhabditis elegans genome using RNAi. Nature 2003, 421(6920):231-237.

25. Maeda I, Kohara Y, Yamamoto M, Sugimoto A: Large-scale analysis of gene function in Caenorhabditis elegans by high-throughput RNAi. Curr Biol 2001, 11(3):171-176.

26. Piano F, Schetter AJ, Mangone M, Stein L, Kemphues KJ: RNAi analysis of genes expressed in the ovary of Caenorhabditis elegans. Curr Biol 2000, 10(24):1619-1622.

27. Piano F, Schetter AJ, Morton DG, Gunsalus KC, Reinke V, Kim SK, Kemphues KJ: Gene clustering based on RNAi phenotypes of ovary-enriched genes in C. elegans. Curr Biol 2002, 12(22):1959-1964.

28. Hodgkin J, Doniach T: Natural variation and copulatory plug formation in Caenorhabditis elegans. Genetics 1997, 146(1):149-164.

29. Kiontke K, Gavin NP, Raynes Y, Roehrig C, Piano F, Fitch DH: Caenorhabditis phylogeny predicts convergence of hermaphroditism and extensive intron loss. Proc Natl Acad Sci USA 2004, 101(24):9003-9008.

30. Baldi C, Cho S, Ellis RE: Mutations in two independent pathways are sufficient to create hermaphroditic nematodes. Science 2009, 326(5955):1002-1005.

31. Grumbling G, Strelets V: FlyBase: anatomical data, images and queries. Nucleic Acids Res 2006, , 34 Database: D484-488.

32. Robinson PN, Kohler S, Bauer S, Seelow D, Horn D, Mundlos S: The Human Phenotype Ontology: a tool for annotating and analyzing human hereditary disease. Am J Hum Genet 2008, 83(5):610-615. 
33. Smith $\mathrm{CL}$, Goldsmith CA, Eppig JT: The Mammalian Phenotype Ontology as a tool for annotating, analyzing and comparing phenotypic information. Genome Biol 2005, 6(1):R7.

34. Sprague J, Bayraktaroglu L, Bradford Y, Conlin T, Dunn N, Fashena D, Frazer K, Haendel M, Howe DG, Knight J, Mani P, Moxon SA, Pich C, Ramachandran S, Schaper K, Segerdell E, Shao X, Singer A, Song P, Sprunger B, Van Slyke CE, Westerfield M: The Zebrafish Information Network: the zebrafish model organism database provides expanded support for genotypes and phenotypes. Nucleic Acids Res 2008, , 36 Database: D768-772.

35. Yamazaki Y, Jaiswal P: Biological ontologies in rice databases. An introduction to the activities in Gramene and Oryzabase. Plant Cell Physiol 2005, 46(1):63-68.

36. Washington NL, Haendel MA, Mungall CJ, Ashburner M, Westerfield M, Lewis SE: Linking human diseases to animal models using ontologybased phenotype annotation. PLOS Biol 2009, 7(11):e1000247.

37. Gkoutos GV, Green EC, Mallon AM, Hancock JM, Davidson D: Using ontologies to describe mouse phenotypes. Genome Biol 2005, 6(1):R8.

38. Mabee PM, Arratia G, Coburn M, Haendel M, Hilton EJ, Lundberg JG, Mayden RL, Rios N, Westerfield M: Connecting evolutionary morphology to genomics using ontologies: a case study from Cypriniformes including zebrafish. J Exp Zool B Mol Dev Evol 2007, 308(5):655-668.

39. Mabee PM, Ashburner M, Cronk Q, Gkoutos GV, Haendel M, Segerdell E, Mungall C, Westerfield M: Phenotype ontologies: the bridge between genomics and evolution. Trends Ecol Evol 2007, 22(7):345-350.

40. Raizen DM, Zimmerman JE, Maycock MH, Ta UD, You YJ, Sundaram MV, Pack Al: Lethargus is a Caenorhabditis elegans sleep-like state. Nature 2008, 451(7178):569-572

41. Smith B, Ceusters W, Klagges B, Kohler J, Kumar A, Lomax J, Mungall C, Neuhaus $F$, Rector AL, Rosse C: Relations in biomedical ontologies. Genome Biol 2005, 6(5):R46.

42. Bodenreider $\mathrm{O}$, Stevens R: Bio-ontologies: current trends and future directions. Brief Bioinform 2006, 7(3):256-274

43. Harris TW, Stein LD: WormBase: methods for data mining and comparative genomics. Methods Mol Biol 2006, 351:31-50.

44. Stein LD, Mungall C, Shu S, Caudy M, Mangone M, Day A, Nickerson E, Stajich JE, Harris TW, Arva A, Lewis S: The generic genome browser: a building block for a model organism system database. Genome Res 2002, 12(10):1599-1610.

45. Cho S, Jin SW, Cohen A, Ellis RE: A phylogeny of caenorhabditis reveals frequent loss of introns during nematode evolution. Genome Res 2004, 14(7):1207-1220

46. Haag ES: Convergent evolution: regulatory lightning strikes twice. Curr Biol 2009, 19(21):R977-979.

47. Clifford R, Lee MH, Nayak S, Ohmachi M, Giorgini F, Schedl T: FOG-2, a novel F-box containing protein, associates with the GLD-1 RNA binding protein and directs male sex determination in the $C$. elegans hermaphrodite germline. Development 2000, 127(24):5265-5276.

48. Schedl T, Kimble J: fog-2, a germ-line-specific sex determination gene required for hermaphrodite spermatogenesis in Caenorhabditis elegans. Genetics 1988, 119(1):43-61.

49. Nayak S, Goree J, Schedl T: fog-2 and the evolution of self-fertile hermaphroditism in Caenorhabditis. PLoS Biol 2005, 3(1):e6.

50. Guo $Y$, Lang $S$, Ellis RE: Independent recruitment of $F$ box genes to regulate hermaphrodite development during nematode evolution. Curr Biol 2009, 19(21):1853-1860.

51. Francis R, Barton MK, Kimble J, Schedl T: gld-1, a tumor suppressor gene required for oocyte development in Caenorhabditis elegans. Genetics 1995, 139(2):579-606

52. Ciche TA, Sternberg PW: Postembryonic RNAi in Heterorhabditis bacteriophora: a nematode insect parasite and host for insect pathogenic symbionts. BMC Dev Biol 2007, 7:101.

53. Lambie EJ, Kimble J: Two homologous regulatory genes, lin-12 and glp-1, have overlapping functions. Development 1991, 112(1):231-240.

54. Kohler S, Schulz MH, Krawitz P, Bauer S, Dolken S, Ott CE, Mundlos C, Horn D, Mundlos S, Robinson PN: Clinical diagnostics in human genetics with semantic similarity searches in ontologies. Am J Hum Genet 2009, 85(4):457-464.

55. Degtyarenko $K$, de Matos $P$, Ennis $M$, Hastings J, Zbinden M, McNaught $A$, Alcantara R, Darsow M, Guedj M, Ashburner M: ChEBI: a database and ontology for chemical entities of biological interest. Nucleic Acids Res 2008, , 36 Database: D344-350.

56. Haendel M, Gkoutos G, Lewis S, Mungall C: Uberon: towards a comprehensive multi-species anatomy ontology. Nature Precedings 2009

57. Malone BM, Perkins AD, Bridges SM: Integrating phenotype and gene expression data for predicting gene function. BMC Bioinformatics 2009 10(Suppl 11):S20.

58. Zhong W, Sternberg PW: Genome-wide prediction of $C$. elegans genetic interactions. Science 2006, 311(5766):1481-1484.

59. McGary KL, Park TJ, Woods JO, Cha HJ, Wallingford JB, Marcotte EM: Systematic discovery of nonobvious human disease models through orthologous phenotypes. Proc Natl Acad Sci USA 2010, 107(14):6544-6549.

60. Dryja TP, Cavenee W, White R, Rapaport JM, Petersen R, Albert DM, Bruns GA: Homozygosity of chromosome 13 in retinoblastoma. $N$ Engl J Med 1984, 310(9):550-553.

61. Lu X, Horvitz HR: lin-35 and lin-53, two genes that antagonize a C. elegans Ras pathway, encode proteins similar to $\mathrm{Rb}$ and its binding protein RbAp48. Cell 1998, 95(7):981-991.

62. Amberger J, Bocchini CA, Scott AF, Hamosh A: McKusick's Online Mendelian Inheritance in Man (OMIM). Nucleic Acids Res 2009, 37 Database: D793-796.

63. Day-Richter J, Harris MA, Haendel M, Lewis S: OBO-Edit-an ontology editor for biologists. Bioinformatics 2007, 23(16):2198-2200.

64. Smith B, Ashburner M, Rosse C, Bard J, Bug W, Ceusters W, Goldberg LJ, Eilbeck K, Ireland A, Mungall CJ, Leontis N, Rocca-Serra P, Ruttenberg A, Sansone SA, Scheuermann RH, Shah N, Whetzel PL, Lewis S: The OBO Foundry: coordinated evolution of ontologies to support biomedical data integration. Nat Biotechnol 2007, 25(11):1251-1255.

65. Gravato-Nobre MJ, Nicholas HR, Nijland R, O'Rourke D, Whittington DE, Yook KJ, Hodgkin J: Multiple genes affect sensitivity of Caenorhabditis elegans to the bacterial pathogen Microbacterium nematophilum. Genetics 2005, 171(3):1033-1045.

66. Brisbin S, Liu J, Boudreau J, Peng J, Evangelista M, Chin-Sang I: A role for C. elegans Eph RTK signaling in PTEN regulation. Dev Cell 2009, 17(4):459-469.

67. Daniels SA, Ailion M, Thomas JH, Sengupta P: egl-4 acts through a transforming growth factor-beta/SMAD pathway in Caenorhabditis elegans to regulate multiple neuronal circuits in response to sensory cues. Genetics 2000, 156(1):123-141.

68. Hannich JT, Entchev EV, Mende F, Boytchev H, Martin R, Zagoriy V, Theumer G, Riezman I, Riezman H, Knolker HJ, Kurzchalia TV: Methylation of the sterol nucleus by STRM-1 regulates dauer larva formation in Caenorhabditis elegans. Dev Cell 2009, 16(6):833-843.

69. Kim K, Sato K, Shibuya M, Zeiger DM, Butcher RA, Ragains JR, Clardy J, Touhara K, Sengupta P: Two chemoreceptors mediate developmental effects of dauer pheromone in C. elegans. Science 2009, 326(5955):994-998

70. Reiner DJ, Ailion M, Thomas JH, Meyer BJ: C. elegans anaplastic lymphoma kinase ortholog SCD-2 controls dauer formation by modulating TGFbeta signaling. Curr Biol 2008, 18(15):1101-1109.

71. Albert PS, Brown SJ, Riddle DL: Sensory control of dauer larva formation in Caenorhabditis elegans. J Comp Neurol 1981, 198(3):435-451.

72. Gupta BP, Johnsen R, Chen N: Genomics and biology of the nematode Caenorhabditis briggsae. WormBook 2007, 1-16.

doi:10.1186/1471-2105-12-32

Cite this article as: Schindelman et al:: Worm Phenotype Ontology: Integrating phenotype data within and beyond the C. elegans community. BMC Bioinformatics 2011 12:32. 